\title{
A Novel Compact Balanced-to-Unbalanced Low-Temperature Co-Fired Ceramic Bandpass Filter With Three Coupled Lines Configuration
}

\author{
Kun-Tzu Chen and Shyh-Jong Chung, Senior Member, IEEE
}

\begin{abstract}
This paper proposes a novel compact balanced-to-unbalanced bandpass filter. Firstly, a pre-design circuit is presented, which is composed of an inductive coupled-line bandpass filter and an out-of-phase capacitive coupled-line bandpass filter. A novel compact circuit with three coupled lines configuration, derived from the pre-design circuit, is then proposed for miniaturizing the balanced-to-unbalanced bandpass filter. A $2.4-\mathrm{GHz}$ multilayer ceramic chip type balanced-to-unbalanced bandpass filter with a size of $2.0 \mathrm{~mm} \times 1.2 \mathrm{~mm} \times 0.7 \mathrm{~mm}$ is developed to validate the feasibility of the proposed structure. The filter is designed by using circuit simulation, as well as full-wave electromagnetic simulation softwares, and fabricated by the use of low-temperature co-fired ceramic technology. The measured results agree quite well with the simulated. According to the measurement results, the maximum insertion loss is $\mathbf{1 . 6 5} \mathrm{dB}$, the maximum in-band phase imbalance is within $3^{\circ}$, and the maximum in-band magnitude imbalance is less than $0.32 \mathrm{~dB}$.
\end{abstract}

Index Terms-Balanced-to-unbalanced bandpass filter, balun, bandpass filter, low-temperature co-fired ceramic (LTCC).

\section{INTRODUCTION}

B ALANCED circuits became more popular due to better gain and second-order linearity, superior spurious response performance, and noise immunity [1]. Therefore, they have been widely used in the microwave systems. In the receiver design, the generality of RF integrated circuits (RFICs) demand balanced input, but RF signals are output from the antenna or filter unbalanced. Thus, a balanced-to-unbalanced component (such as a balun) is needed that can handle unbalanced input and balanced output.

The balun is a passive electronic device that converts a balanced transmission signal into an unbalanced signal and vice versa. In a balun, one pair of terminals is balanced, i.e., the currents are equal in magnitude and opposite in phase. The other pair of terminals is unbalanced; one side is connected to an electrical ground and the other carries the signal. Recently, there have been many papers that have investigated configurations of the balun. Compact designs to miniaturize the balun were reported in the literature [2]-[6]. The baluns have good transmis-

Manuscript received December 31, 2007; revised March 27, 2008. First published June 20, 2008; last published July 9, 2008 (projected). This work was supported in part by the National Science Council, R.O.C., under Contract NSC 96-2752-E009-003-PAE.

The authors are with the Department of Communication Engineering, National Chiao Tung University, Hsinchu 30050, Taiwan, R.O.C. (e-mail: sjchung@cm.nctu.edu.tw).

Digital Object Identifier 10.1109/TMTT.2008.926196

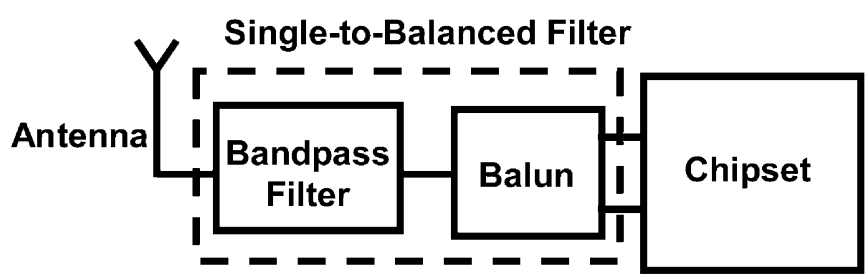

Fig. 1. Typical architecture of RF front-end module. The bandpass filter and the balun can be replaced by a balanced-to-unbalanced bandpass filter.

sion performance, but usually with poor out-band suppression. Therefore, a bandpass filter is often used next to the balun.

The bandpass filter is also one of the important components in the RF frond-end module, which is a device that passes the signals of frequencies within a certain range and rejects (attenuates) unwanted signals outside that range. To improve the filter performance, out-band suppression has been proposed in earlier works [7]-[10]. Most of the configurations are with unbalanced input and output.

As the portable communication products advance toward small size, light weight, and high performance, development of a compact design of components in the RF front-end module is important and needed. The low-temperature co-fired ceramic (LTCC) provides a 3-D multilayer carrier for RF circuitry, and thus, is a good choice for realizing compact components.

Fig. 1 shows a typical architecture of an RF front-end module, which includes an antenna, a bandpass filter, a balun, and a transceiver chip set. The balun and bandpass filter are often cascaded next to each other. Therefore, it is easy and convenient to integrate them together by using the LTCC technology. In [11], an LTCC substrate embedded with a combline bandpass filter and a Marchand balun for Bluetooth application was investigated. In the design, the bandpass filter and the balun were integrated directly, which would occupy too much circuitry space. Therefore, to reduce the size and improve the performance (e.g., insertion loss), it is better to integrate them together in an LTCC substrate, and simplify the circuit scheme by removing common circuit elements. Earlier works [12]-[14] have proposed balanced-to-unbalanced bandpass filters that integrate a conventional bandpass filter and a balun with one of the transmission lines used both in the bandpass filter and balun. One transmission line was left out in their designs. A balanced-to-unbalanced bandpass filter yielded by imaging a typical two-port filter at a virtual ground was proposed in [15]. Moreover, [16] proposed a balanced-to-unbalanced bandpass filter based on a transformer-type balun.

Most of the balanced-to-unbalanced bandpass filters have a shortcoming of bulky size due to the complex configuration. 
In this study, a more compact configuration and miniature of the second-order balanced-to-unbalanced bandpass filter is proposed. For size minimization, the balanced-to-unbalanced bandpass filter is designed and demonstrated using LTCC multilayer technology.

\section{Pre-Design of a BaLANCED-TO-UnBalAnCED BANDPASS FILTER}

Fig. 2(a) and (b) indicates two types of bandpass filters, one with an inductive coupled line, and the other with a capacitive coupled line [17]. The phases of these two bandpass filters change often as resonances occur. Moreover, these two bandpass filters exhibit a $180^{\circ}$ out-of-phase response in the entire band because of opposite coupling directions at the output port. On the other hand, the transmission properties of these two bandpass filters can be designed with the same performance at the operation frequency band by using appropriate values of the elements.

Connecting the inductive coupled-line bandpass filter and capacitive coupled-line bandpass filter in a shunt configuration produces a new circuit of a balanced-to-unbalanced bandpass filter, as shown in Fig. 2(c). The operation frequency band is determined by the shunt capacitors $\left(C_{2+}, C_{2-}, C_{3+}\right.$, and $C_{3-}$ ) and the lengths of the coupled transmission lines $\left(S L_{1+}, S L_{1-}, S L_{2+}\right.$, and $\left.S L_{2-}\right)$. The input impedance can also be fine tuned by series capacitors $\left(C_{1+}, C_{1-}, C_{4+}\right.$, and $C_{4-}$ ). In fact, the matching network in this circuit should be a series-shunt matching network, in which the shunt elements can be absorbed by parallel capacitors $\left(C_{2+}, C_{2-}, C_{3+}\right.$, and $\left.C_{3-}\right)$.

In Fig. 2(c), obviously port $P_{1}$ is an unbalanced input, which is a shunt configuration of the input ports of inductive coupled-line bandpass filter (port $P_{1+}$ ) and capacitive coupled-line bandpass filter (port $P_{1-}$ ) in Fig. 2(a) and (b), respectively. The balanced port also consists of port $P_{2}$ and port $P_{3}$, corresponding to the output ports of inductive coupled-line and capacitive coupled-line bandpass filters. If the input impedance of port $P_{1+}$ and port $P_{1-}$ are the same, then the input impedance of port $P_{1}$ is half that of port $P_{1+}$ (or port $P_{1-}$ ). While an unbalanced signal is fed to port $P_{1}$, its power will be equally divided into the inductive coupled-line and capacitive coupled-line filters through the capacitors $C_{1+}$ and $C_{1-}$. It is then also coupled to the balanced port by way of an inductive coupled line and capacitive coupled line. Due to the different coupling mechanism between them, the output signals at port $P_{2}$ and port $P_{3}$ reveal out-of-phase. Obviously this pre-design circuit can convert an unbalanced signal input at port $P_{1}$ to a balanced output between port $P_{2}$ and port $P_{3}$, and vice versa. Furthermore, all the resonators can be designed in the same resonant frequency. Therefore, this pre-design circuit will transmit the energy at the specific resonant frequency band and restrain signals at the other frequency bands like the conventional bandpass filter. It is quite obvious that the unbalanced input signal becomes the balanced output through the resonators; furthermore each path has the same out-band rejection. It is apparently that this pre-design circuit has the characteristics of the balun and the bandpass filter at the same time. It can thus be regarded as a simple and directly structure of the balanced-to-unbalanced bandpass filter.

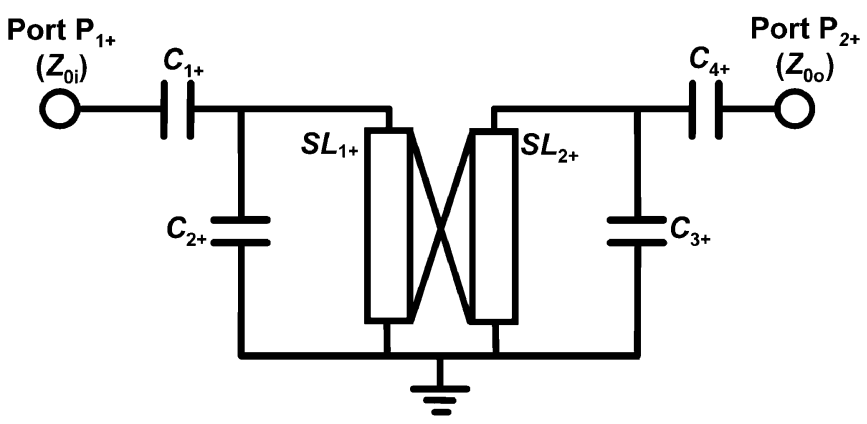

(a)

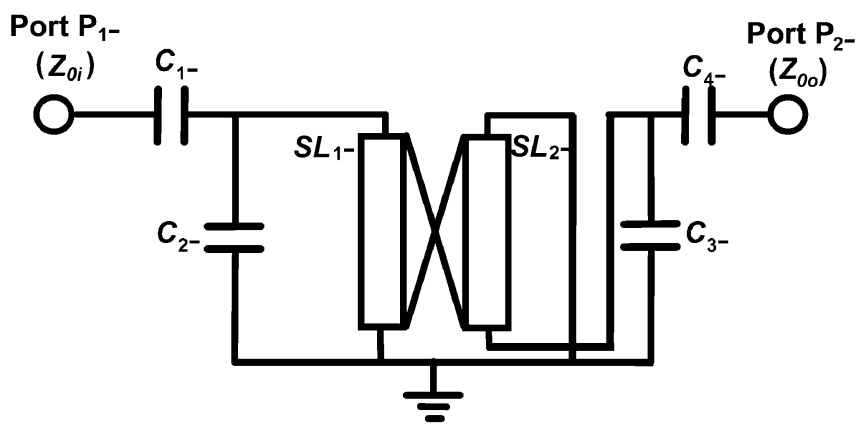

(b)

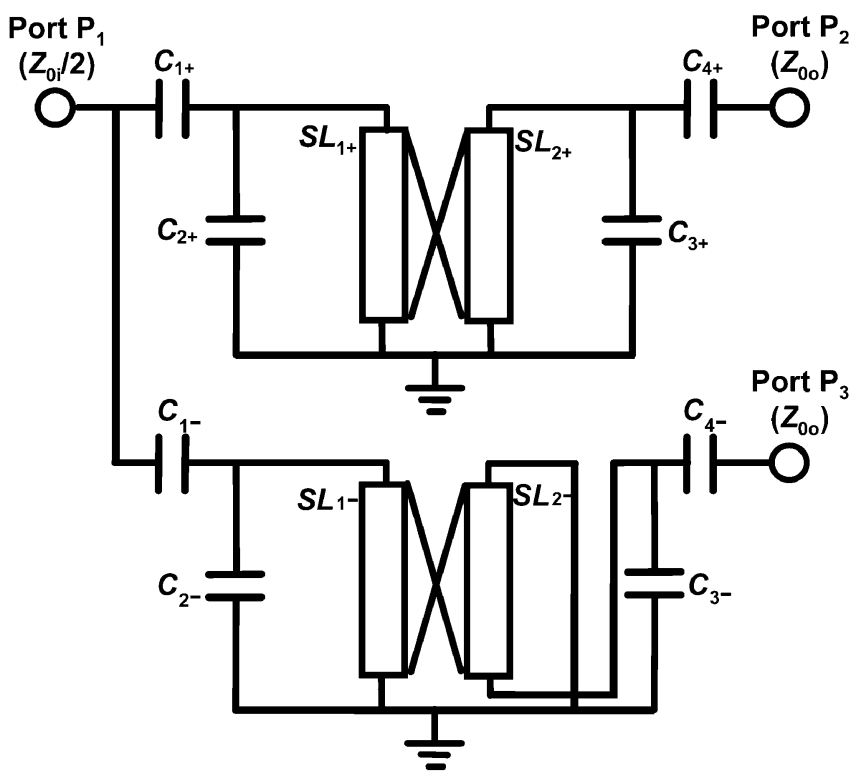

(c)

Fig. 2. (a) Inductive coupled-line bandpass filter. (b) Capacitive coupled-line bandpass filter. (c) Pre-design circuit architecture of the proposed balanced-tounbalanced bandpass filter.

The proposed pre-design circuit of the balanced-to-unbalanced bandpass filter can be easily synthesized from conventional bandpass filters. Firstly, a second-order bandpass filter of input impedance $Z_{0 i}$ and output impedance $Z_{0 o}$ is designed using a filter synthesis method like those in [18]. Then convert the inverter between resonators to a mutual inductance like that in the inductive coupled-line bandpass filter by using formulas such as those in [6]. After that, a capacitive coupled-line bandpass filter can be implemented with the same element values, but using out-of-phase output. Finally, connect these bandpass filters directly with shunt configuration, as 


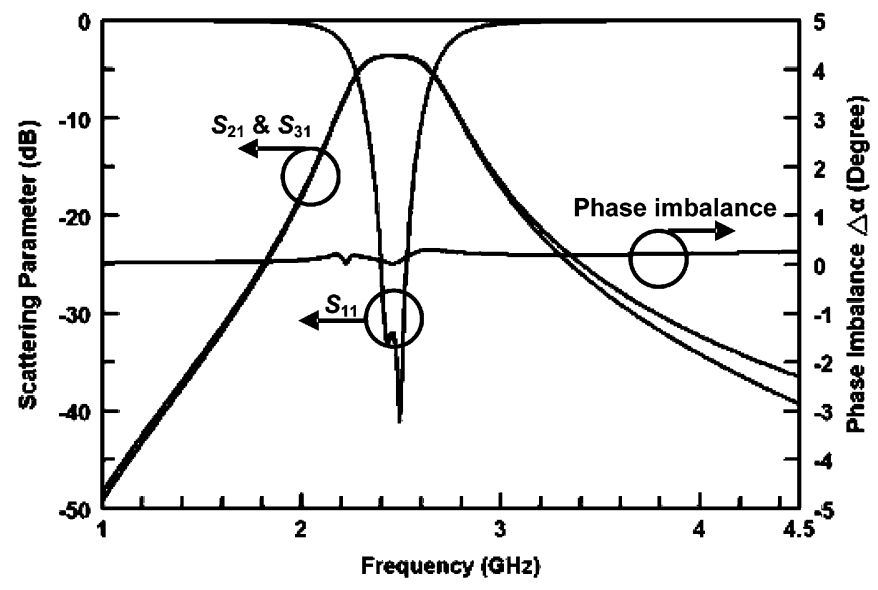

Fig. 3. Scattering parameters and the magnitude and phase imbalances of the pre-design balanced-to-unbalanced bandpass filter using ideal elements.

shown in Fig. 2(c), forming a balanced-to-unbalanced bandpass filter. The accomplished filter would have an unbalanced port (port $P_{1}$ ) with an impedance of $Z_{0 i} / 2$ and a balanced output port (port $P_{2}$ and port $P_{3}$ ) with an impedance of $2 Z_{0 o}$.

A 2.4-GHz balanced-to-unbalanced bandpass filter is simulated from this pre-design circuit by using the circuit simulator Microwave Office [19]. Fig. 3 shows the results. In the simulation, a circuit substrate with a dielectric constant of 7.8 , loss tangent of 0.002 , and thickness of $0.7 \mathrm{~mm}$ are used. The direct current blocking capacitors $C_{1+}, C_{1-}, C_{4+}$, and $C_{4-}$ are equal to $2.0 \mathrm{pF}$, and the grounding capacitors $C_{2+}, C_{2-}, C_{3+}$, and $C_{3-}$ are $4.74 \mathrm{pF}$. The physical lengths of the transmission lines $S L_{1+}, S L_{1-}, S L_{2+}$, and $S L_{2-}$ are the same and equal to $1.69 \mathrm{~mm}$, and their widths are $0.15 \mathrm{~mm}$. The spacing between $S L_{1+}$ and $S L_{1-}$ is $0.21 \mathrm{~mm}$ and that between $S L_{2+}$ and $S L_{2-}$ is $0.215 \mathrm{~mm}$. Input impedance of the unbalanced port (port $P_{1}$ ) is $25 \Omega\left(Z_{0 i} / 2\right)$ and is $100 \Omega\left(2 Z_{0 o}\right)$ for the balanced port (ports $P_{2}$ and port $P_{3}$ ). According to the simulation results, the scattering parameters $S_{21}$ and $S_{31}$ are approximately $-3.7 \mathrm{~dB}$ in the frequency band from 2.4 to $2.5 \mathrm{GHz}$, which corresponds to an insertion loss of $0.7 \mathrm{~dB}$ at the balanced port. The magnitude imbalance and the phase imbalance at this port are within $0.1 \mathrm{~dB}$ and $0.5^{\circ}$, respectively. Note that the phase imbalance $\Delta \alpha$ is defined as

$$
\Delta \alpha=\angle S_{21}-\angle S_{31}-180^{\circ} .
$$

\section{BALANCED-TO-UNBALANCED BANDPASS FILTER USING THREE COUPLED LINES}

\section{A. Circuit Model}

The pre-design circuit of the balanced-to-unbalanced bandpass filter is workable as demonstrated. However, this circuit still has two major problems. First, the inductive coupled-line and capacitive coupled-line must have proper shielding to reduce the cross-coupled interactions between the two sets of transmission lines. This can be done by using a ground layer in between them. However, it will cause a new problem, i.e., that the number of LTCC layers will increase to reduce the parasitic capacitors between the ground layer and the coupled

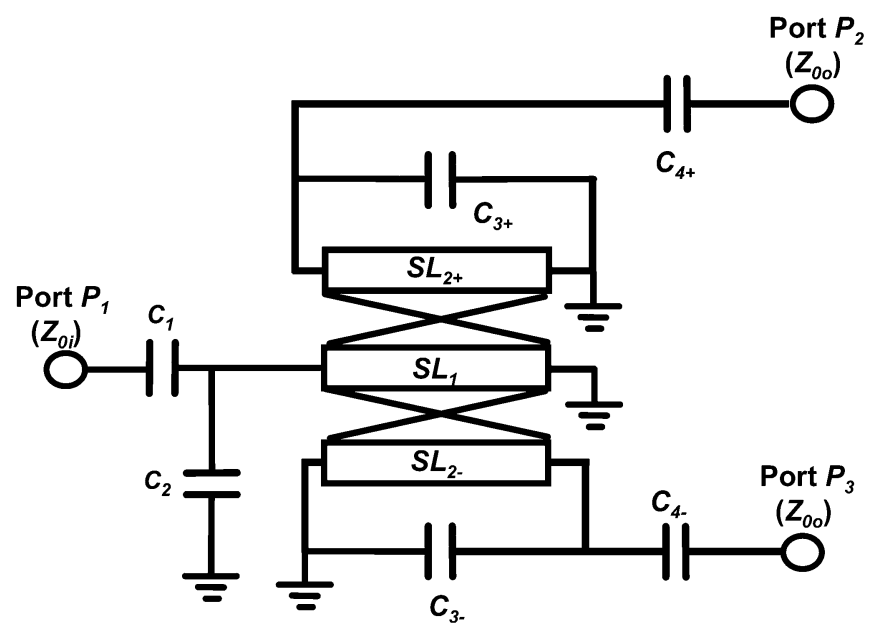

Fig. 4. Compact architecture of the balanced-to-unbalanced bandpass filter merged with the inductive coupled-line and capacitive coupled-line configuration.

transmission lines, which also means a higher fabrication cost. The second problem of the pre-design circuit is its bulky size since the pre-design circuit consists of two filters, each with two resonators. Therefore, reducing the number of resonators to downsize the balanced-to-unbalanced bandpass filter makes sense.

Fig. 4 shows a compact circuit solution to these problems, which is an improvement of the pre-design circuit. In this circuit, the inductive coupled-line and capacitive coupled-line are replaced by the three coupled lines to solve the shielding problem. Two of the resonators in the pre-design circuit are also merged into one, resulting in a reduction of the number of resonators and, thus, the filter size.

All elements of Fig. 4 can correspond to those of the pre-design circuit shown in Fig. 2(c). Capacitor $C_{1}$ in Fig. 4 is obtained by combining capacitors $C_{1+}$ and $C_{1-}$ in Fig. 2(c). In the same way, capacitors $C_{2+}$ and $C_{2-}$ in Fig. 2(c) are merged into capacitor $C_{2}$ in Fig. 4, and transmission lines $S L_{1+}$ and $S L_{1-}$ in Fig. 2(c) are replaced by transmission line $S L_{1}$ in Fig. 4. The balanced-to-unbalanced bandpass filter has three capacitors $\left(C_{1}, C_{4+}\right.$, and $\left.C_{4-}\right)$ for direct current blocking and impedance matching. On the other hand, there are only three resonators in this circuit, each with a grounding capacitor $\left(C_{2}, C_{3+}\right.$, or $\left.C_{3-}\right)$ and a stripline $\left(S L_{1}, S L_{2+}\right.$, or $\left.S L_{2-}\right)$. These resonators constitute the passband of the filter. Furthermore, the transmission lines $S L_{1}$ and $S L_{2}$ form the inductive coupled-line, and further, $S L_{1}$ and $S L_{2-}$ form the capacitive coupled-line. The out-of-phase property of the inductive coupled-line and capacitive coupled-line is still used in this circuit to provide the phase shifting. Like the pre-design circuit, port $P_{1}$ is an unbalanced input port, and the balanced port is composed of ports $P_{2}$ and $P_{3}$.

In order to verify the performance of the proposed circuit, a 2.4-GHz balanced-to-unbalanced bandpass filter is designed from this new structure. Fig. 5 shows the circuit simulation results. In this simulation, a circuit substrate with a dielectric constant of 7.8, loss tangent of 0.002 , and thickness of $0.572 \mathrm{~mm}$ are used. The direct current blocking capacitors $C_{1}, C_{4+}$, and $C_{4-}$ are equal to $2.0,1.47$, and $1.47 \mathrm{pF}$, respectively. The grounding 


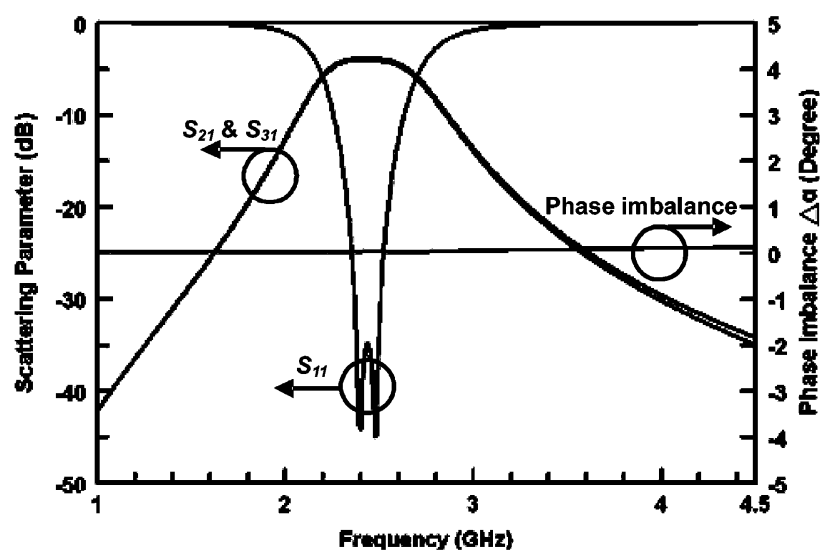

Fig. 5. Scattering parameters and the magnitude and phase imbalances of the proposed balanced-to-unbalanced bandpass filter from circuit simulation.

capacitors $C_{2}, C_{3+}$, and $C_{3-}$ are also equal to $4.59,4.5$, and $4.5 \mathrm{pF}$, respectively. The physical lengths of the transmission lines $S L_{1}, S L_{2+}$, and $S L_{2-}$ are equal to $1.56 \mathrm{~mm}$, and their widths are $0.1 \mathrm{~mm}$. The spacing between each coupled transmission line is $0.26 \mathrm{~mm}$. All ports in this design have the same input impedance of $50 \Omega$, which means a $50-\Omega$ unbalanced port and a $100-\Omega$ balanced port. All the resonators have the same resonant frequency at $2.44 \mathrm{GHz}$. Therefore, the magnitude response will have two poles near $2.44 \mathrm{GHz}$. According to the simulation results in Fig. 5, the imbalance transmission performance between $S_{21}$ and $S_{31}$ is within $0.1 \mathrm{~dB}$ in the passband, and the phase imbalance is very close to $0^{\circ}$ from 1 to $4.5 \mathrm{GHz}$. The maximum insertion loss and minimum return loss in the passband are 1.0 and $32 \mathrm{~dB}$, respectively. The results are quite the same as those of the pre-design circuit, although the new filter has a more compact structure.

\section{B. Transformation of Circuit Model to 3-D Structure}

The architecture of the proposed balanced-to-unbalanced bandpass filter is composed of the capacitors and the coupled transmission lines. The capacitors can be implied in the LTCC substrate by metal-insulator-metal or vertical interdigitated capacitor with the parallel-plate capacitor formula of $C=\varepsilon A / d$, where $\varepsilon$ is the permittivity of the substrate, $A$ is the overlapping area of the two plates, and $d$ is the spacing between the two plates. To cover the fabrication tolerance, one of the plates should be larger than the other with at least $50 \mu \mathrm{m}$ in each side when designing a parallel-plate capacitor. Actually, due to the fringing field effect, the area of the plates will need to be fine tuned by electromagnetic (EM) simulation software. In addition, the physical size of the coupled transmission line depends on the circuit simulation result. However, it also need to be fine tuned by EM simulation software. On the other hand, due to the process limit, the minimum linewidth, minimum spacing between lines, and minimum via diameter are 0.1 , 0.075 , and $0.1 \mathrm{~mm}$, respectively.

Before layout, some issues should be considered. First, for the electromagnetic interference (EMI) shielding, the first and last layers in the LTCC layout must be full of metal and connected to the electrical ground. The second issue is that to reduce the ohmic loss and the parasitic capacitances produced be-

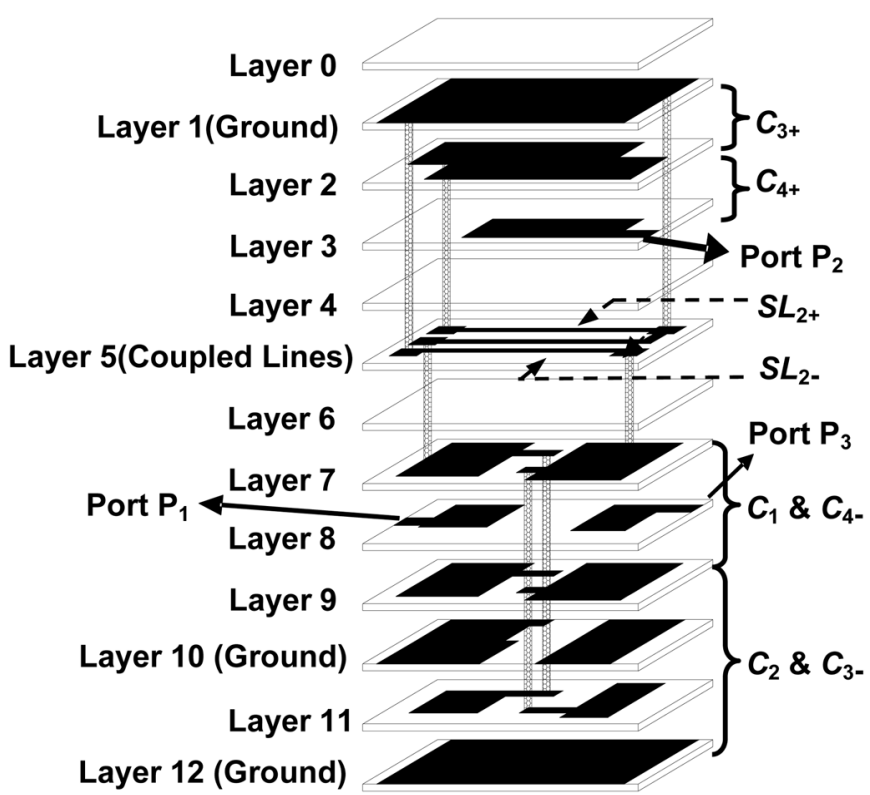

Fig. 6. 3-D LTCC layout for EM simulation of a 2.4-GHz balanced-to-unbalanced bandpass filter with size of $2.0 \mathrm{~mm} \times 1.2 \mathrm{~mm} \times 0.7 \mathrm{~mm}$.

tween coupled transmission lines (or coupled inductors) and the ground layer, the transmission lines are better to be placed on the middle layer of the LTCC so as to have a maximum distance to the ground. Moreover, it is better to use edge coupling instead of broadside coupling, between the coupled lines, to decrease the amount of LTCC layers. The third issue is that since the balanced port consists of port $P_{2}$ and port $P_{3}$, these two ports should be located as close as possible and symmetrically to the unbalanced port (port $P_{1}$ ) to ensure the balance.

The proposed balanced-to-unbalanced bandpass filter was fabricated by a nonshrinkage low-temperature co-fired ceramic (NS-LTCC) fabrication process. The component size is $2.0 \mathrm{~mm} \times 1.2 \mathrm{~mm} \times 0.7 \mathrm{~mm}$ and designed using 13 layers of the NS-LTCC. The thickness of each layer is $0.035 \mathrm{~mm}$, except for layers 3-6, which is $0.096 \mathrm{~mm}$. The material of LTCC used here is NEG MLS-1000, which has a dielectric constant of 7.8 and loss tangent of 0.002 . The metal thickness of each layer is $0.008 \mathrm{~mm}$.

\section{LTCC Layout and EM Simulation}

Fig. 6 illustrates the layout of the balanced-to-unbalanced bandpass filter. Layer 0 is used to keep air off of the metal on layer 1. This LTCC layout has three ground layers on layers 1,10 , and 12 , which are connected for equal potential by side electrodes. These side electrodes should be connected to the ground of the evaluation board. Port $P_{1}$ and port $P_{3}$ are located on layer 8 , and port $P_{2}$ on layer 3 . The metals on layers 7 , 9 , and 11 have equaled potential by vertically connecting vias. $C_{1}, C_{2}, C_{3-}$, and $C_{4-}$ are made by vertical interdigitated capacitors. The metals on layers 7-9 produce $C_{1}$ and $C_{4-}$, and layers 9-12 produce $C_{2}$ and $C_{3-}$. The resonator capacitor $C_{3+}$ and direct current blocking capacitor $C_{4+}$ are made by metal-insulator-metal capacitors. The metals on layers 2 and 3 produce $C_{4+}$. Resonator capacitor $C_{3+}$ is formed between layers 1 and 2. Layer 5 is used for the three coupled lines with edge coupling. 


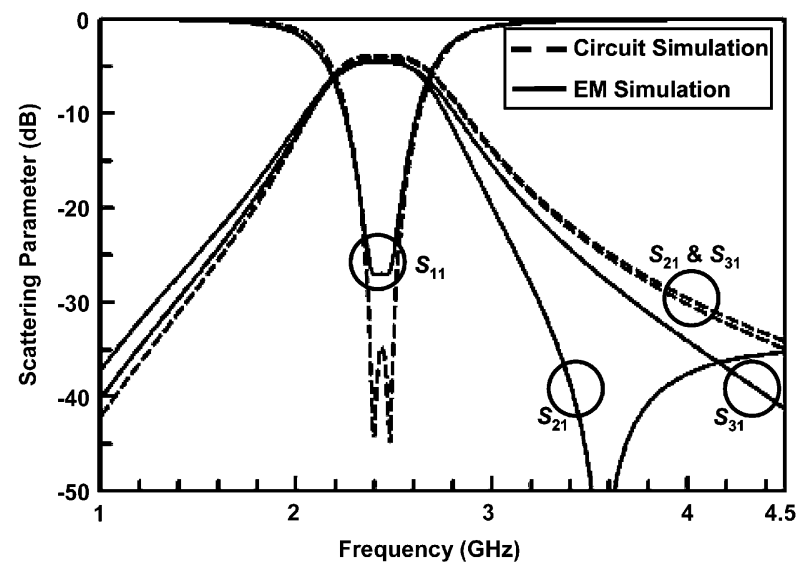

(a)

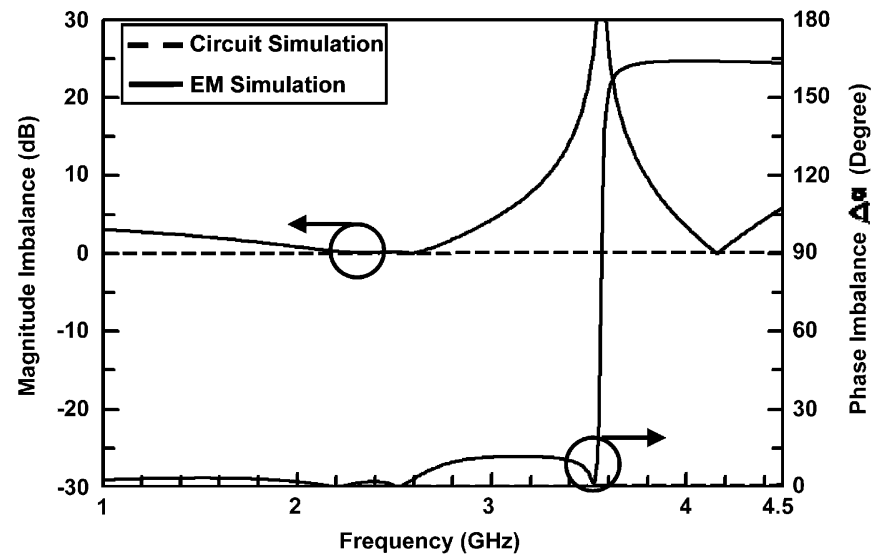

(b)

Fig. 7. Circuit simulation and EM simulation results. (a) Three-port scattering parameters. (b) Phase and magnitude imbalance responses.

The ideal circuit simulations by Applied Wave Research Inc.'s circuit simulator Microwave Office and EM simulations by Ansoft's full-wave commercial package High Frequency Structure Simulator (HFSS) [20] for the design are presented in Fig. 7. The dashed lines indicate the results from the circuit simulation and the solid lines denote EM simulation results.

Both simulation results in Fig. 7(a) match quite well, expect near $3.5 \mathrm{GHz}$, where a transmission zero appears on the inductive coupled-line path $\left(S_{21}\right)$ for the EM simulations. This is due to the parasitic coupling between the metal on layer 3 and the transmission line $S L_{1}$ on layer 5 . The coupling produces a parasitic capacitor and provides a feedback path between port $P_{1}$ and port $P_{2}$, thus causing a transmission zero near $3.5 \mathrm{GHz}$. It is seen from Fig. 7(a) that in the passband from 2.4 to $2.5 \mathrm{GHz}$, the maximum insertion losses $\left(1 / S_{21}\right.$ and $\left.1 / S_{31}\right)$ are $4.6 \mathrm{~dB}$, and the minimum return loss $\left(1 / S_{11}\right)$ is $24.5 \mathrm{~dB}$. The results shown in Fig. 7(b) also indicate that the magnitude imbalance and phase imbalance in the passband are within $0.2 \mathrm{~dB}$ and $2^{\circ}$, respectively.

\section{MEASUREMENT RESULTS}

The fabricated LTCC balance filter is mounted on an evaluation board and measured using Agilent's ENA (E5071B) [21]. The E5071B has four-port measurement capability, and it has built-in functions to provide scattering parameter measurements

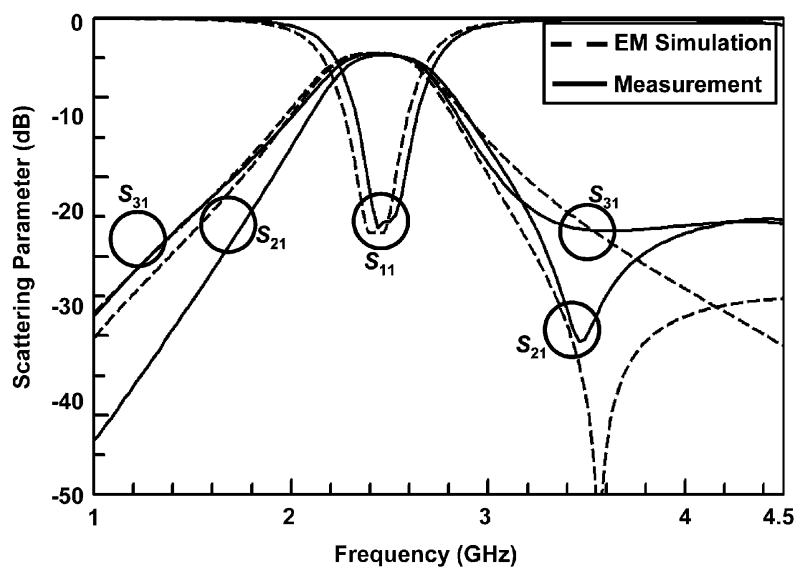

(a)

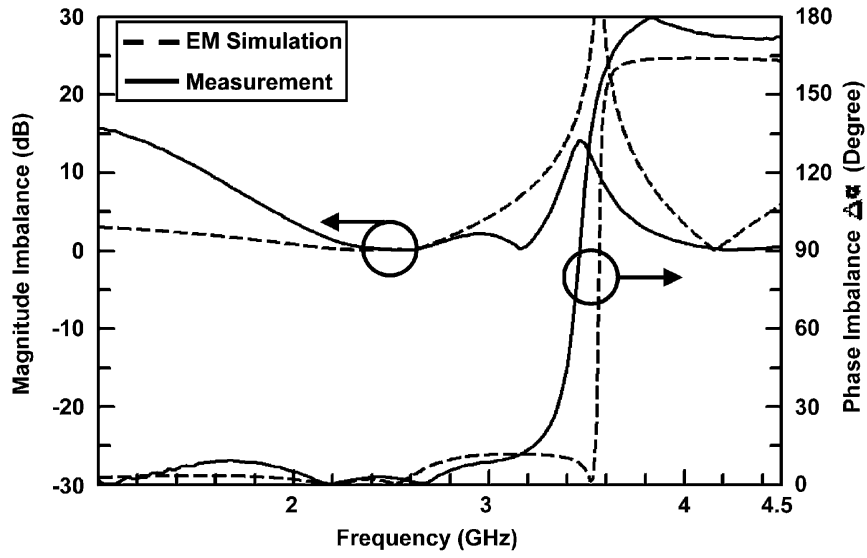

(b)

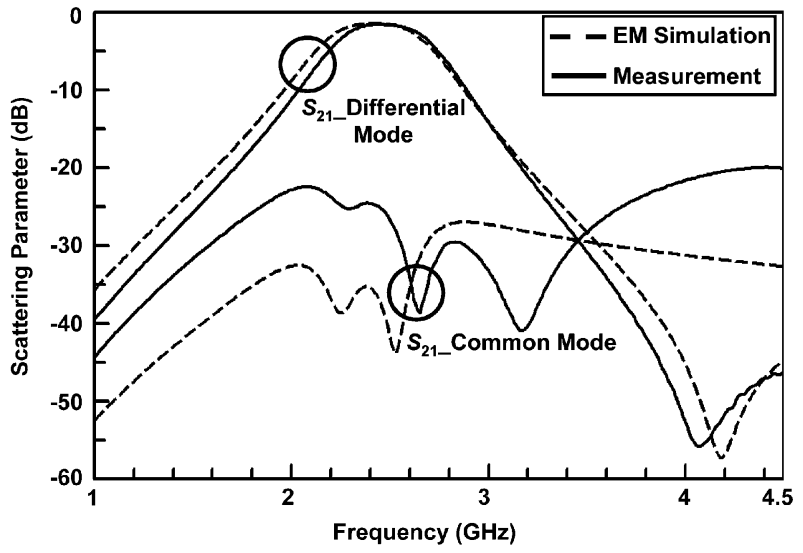

(c)

Fig. 8. Measurement and EM simulation results of the fabricated LTCC 50- $\Omega$ : $100-\Omega$ balanced-to-unbalanced bandpass filter. (a) Three-port scattering parameters. (b) Magnitude and phase imbalance characteristics. (c) Differential- and common-mode transmission characteristics.

in the differential and common modes without any external circuit or calculation. It is thus suitable for the measurement of the balanced-to-unbalanced bandpass filter. Before the measurement of the scattering parameters, the thru-reflect-line (TRL) technique is carefully used for calibration with reference planes at the LTCC side electrodes of the three ports.

Both the EM simulation and measurement results are shown and compared in Fig. 8 with EM simulation results denoted via the dashed line and measurement results denoted via the solid line. The measured scattering parameters shown in Fig. 8(a) 
TABLE I

COMPARISON OF THE PERFORMANCES AND SIZES OF REPORTED 2.4-GHz BALANCED-TO-UNBALANCED BANDPASS FILTERS

\begin{tabular}{|l|l|l|l|}
\hline & $\begin{array}{l}\text { Insertion } \\
\text { Loss }(\mathrm{dB})\end{array}$ & $\begin{array}{l}\text { Mag.(dB)/Phase } \\
(\text { degree) Imbalances }\end{array}$ & $\begin{array}{l}\text { Component } \\
\text { size }\left(\mathrm{mm}^{3}\right)\end{array}$ \\
\hline This work & 1.65 & $0.32 / 3.0$ & $2.0 \times 1.2 \times 0.7$ \\
\hline Ref[12] & 1.8 & $0.46 / 2.2$ & $2.5 \times 2.0 \times 1.0$ \\
\hline Ref[13] & --- & $0.2 / 2$ & $4.15 \times 4.7 \times 0.8$ \\
\hline Ref[14] & --- & $0.3 / 1$ & $4.15 \times 4.7 \times 0.8$ \\
\hline Ref[15] & 1.88 & --- & $2.5 \times 2.0 \times 1.0$ \\
\hline
\end{tabular}

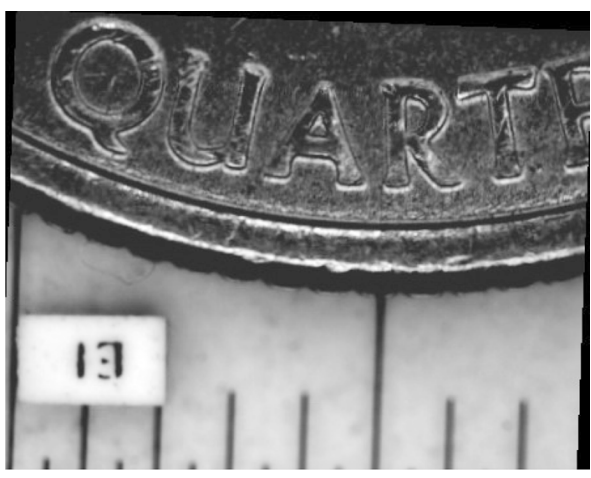

Fig. 9. LTCC balanced-to-unbalanced bandpass filter (with size of $2.0 \mathrm{~mm} \times 1.2 \mathrm{~mm} \times 0.7 \mathrm{~mm}$.)

agree well with the simulation ones. Two transmission poles in the passband can be observed from $S_{11}$ curves with a minimum return loss of $22 \mathrm{~dB}$. The insertion losses $\left(1 / S_{21}\right.$ and $\left.1 / S_{31}\right)$ in the passband at the two output ports are both better than $4.7 \mathrm{~dB}$. A transmission zero of $S_{21}$ near $3.5 \mathrm{GHz}$ was measured, as predicated from the EM simulation. Fig. 8(b) shows the magnitude and phase imbalances of the balanced-to-unbalanced bandpass filter. It is seen that the phase difference between port $P_{2}$ and port $P_{3}$ is near $180^{\circ}$ before the first transmission zero occurred on $S_{21}$ near $3.5 \mathrm{GHz}$, and after this transmission zero, the phase difference changes from out-of-phase to in-phase (i.e., the imbalance phase is $180^{\circ}$ ). The measured magnitude and phase imbalances in the passband are also less than $0.32 \mathrm{~dB}$ and $3^{\circ}$, respectively. According to the measured results, $S_{21}$ and $S_{31}$ have good balanced performance in the passband. Fig. 8(c) shows the transmission performance of the differential and common modes. In the passband, the maximum insertion loss of the differential mode is $1.65 \mathrm{~dB}$, and the minimum insertion loss of the common mode is $24 \mathrm{~dB}$. For the differential mode, there is a transmission zero near $4.2 \mathrm{GHz}$, as can be observed from both the EM simulation and measurement results. The cause of this transmission zero can be explained from the behaviors of the scattering parameters $S_{21}$ and $S_{31}$ after the transmission zero of $S_{21}$ near $3.5 \mathrm{GHz}$, where the output phases change to in-phase. As is seen from Fig. 8(a), the magnitudes of $S_{21}$ and $S_{31}$ are close to each other after the transmission zero and become equal near $4.2 \mathrm{GHz}$. This means that two output signals at port $P_{2}$ and port $P_{3}$ are with equal phase and magnitude at $4.2 \mathrm{GHz}$, thus resulting in the differential mode transmission zero. This transmission zero may reject the harmonic of the local oscillator signal with a frequency near $2.1 \mathrm{GHz}$ by at least $50 \mathrm{~dB}$.

Table I compares the performance and size of the proposed LTCC balanced-to-unbalanced bandpass filter to those of other related studies.
Fig. 9 shows a photograph of the fabricated LTCC balanced-to-unbalanced bandpass filter.

\section{CONCLUSION}

This paper has proposed a novel miniaturized balanced-to-unbalanced bandpass filter that is composed of the inductive coupled-line bandpass filter and the capacitive coupled-line bandpass filter. A balanced-to-unbalanced bandpass filter with an in-band from 2.4 to $2.5 \mathrm{GHz}$ was fabricated and measured to verify the design in a compact size using the LTCC technology. It not only has a low insertion loss (better than $1.65 \mathrm{~dB}$ ), but also has good balanced performances in the passband. As revealed from the measurement, the magnitude imbalance is lower than $0.32 \mathrm{~dB}$, and the phase imbalance is smaller than $3^{\circ}$ in the passband. A finite transmission zero near $4.2 \mathrm{GHz}$ in the differential mode helps to reject the harmonic of the local oscillator signal. The measurement results agree well with those obtained from the full-wave EM simulation. Finally, the good performances, as well as the compact configuration, demonstrate the feasibility of the proposed balanced-to-unbalanced bandpass filter.

\section{ACKNOWLEDGMENT}

The authors are grateful to the SENTEC E\&E Company Ltd., Taoyuan, Taiwan, R.O.C., for nonshrinkage LTCC fabrication. The authors also express much appreciation to W.-B. Teng, SENTEC E\&E Company Ltd., for helpful suggestion. The reviewers of this paper are also appreciated for their valuable comments and suggestions.

\section{REFERENCES}

[1] S. Belkin, "Differential circuit characterization with two-port $S$-parameters," IEEE Micro., pp. 86-99, 2006.

[2] C. M. Tsai and K. C. Gupta, "CAD procedures for planar re-entrant type couplers and three-line baluns,", in IEEE MTT-S Int. Microw. Symp. Dig., 1993, pp. 1013-1016.

[3] C. Cho and K. C. Gupta, "A new design procedure for single-layer and two-layer three-line baluns," IEEE Trans. Microw. Theory Tech., vol. 46, no. 12, pp. 2514-2519, Dec. 1998.

[4] K. S. Ang, Y. C. Leong, and C. H. Lee, "Analysis and design of miniaturized lumped-distributed impedance-transforming baluns," IEEE Trans. Microw. Theory Tech., vol. 51, no. 3, pp. 1009-1017, Mar. 2003.

[5] R. Kravchenko, K. Markov, D. Orlenko, G. Sevskiy, and P. Heide, "Implementation of a miniaturized lumped-distributed balun in balanced filtering for wireless applications," in Proc. Eur. Microw. Conf., 2005, pp. 1303-1306

[6] B. H. Lee, D. S. Park, S. S. Park, and M. C. Park, "Design of new three-line balun and its implementation using multilayer configuration," IEEE Trans. Microw. Theory Tech., vol. 54, no. 6, pp. 1405-1414, Jun. 2006.

[7] L. K. Yeung and K.-L. Wu, "A compact second-order LTCC bandpass filter with two finite transmission zeros," IEEE Trans. Microw. Theory Tech., vol. 51, no. 2, pp. 337-341, Feb. 2003.

[8] C.-W. Tang, Y.-C. Lin, and C.-Y. Chang, "Realization of transmission zeros in combline filters using an auxiliary inductively coupled ground plane," IEEE Trans. Microw. Theory Tech., vol. 51, no. 10, pp. 2112-2118, Oct. 2003.

[9] C.-F. Chang and S.-J. Chung, "Bandpass filter of serial configuration with two finite transmission zeros using LTCC technology," IEEE Trans. Microw. Theory Tech., vol. 53, no. 7, pp. 2383-2388, Jul. 2005.

[10] Y.-S. Lin, C.-H. Wang, C.-H. Wu, and C. H. Chen, "Novel compact parallel-coupled microstrip bandpass filters with lumped-element K-inverters," IEEE Trans. Microw. Theory Tech., vol. 53, no. 7, pp. 2324-2328, Jul. 2005.

[11] D.-W. Yoo, E.-S. Kim, and S.-W. Kim, "A balance filter with DC supply for Bluetooth module," in Proc. Eur. Microw. Conf., 2005, pp. $1239-1242$ 
[12] M. C. Park, B. H. Lee, and D. S. Park, "A laminated balance filter using LTCC technology," in Proc. Asia-Pacific Microw. Conf., 2005, pp. 4-7.

[13] L. K. Yeung and K.-L. Wu, "An integrated RF balanced-filter with enhanced rejection characteristics," in IEEE MTT-S Int. Microw. Symp. Dig., 2005, pp. 713-716.

[14] L. K. Yeung and K.-L. Wu, "An LTCC balanced-to-unbalanced extracted-pole bandpass filter with complex load," IEEE Trans. Microw. Theory Tech., vol. 54, no. 4, pp. 1512-1518, Apr. 2006.

[15] C.-C. Chuang and C. L. Wang, "Design of three-pole single-to-balanced bandpass filters," in Eur. Microw. Conf., 2006, pp. 1193-1196.

[16] S. Sakhnenko, K. Markov, D. Orlenko, A. Yatsenko, B. Vorotnikov, G. Sevskiy, and P. Heide, "LTCC balanced filter based on a transformer type balun for WLAN 802.11 a application," in Eur. Microw. Conf., 2007, pp. 434-437.

[17] K.-C. Lin, C.-F. Chang, M.-C. Wu, and S.-J. Chung, "Dual-bandpass filters with serial configuration using LTCC technology," IEEE Trans. Microw. Theory Tech., vol. 54, no. 6, pp. 2321-2328, Jun. 2006.

[18] G. L. Matthaei, L. Young, and E. M. T. Jones, Microwave Filters, Impedance Matching Networks and Coupling Structures. New York: McGraw-Hill, 1980.

[19] Microwave Office. Appl. Wave Res. Inc., El Segundo, CA, 2002.

[20] HFSS. Ansoft Corporation, Pittsburgh, PA, 2003.

[21] "Agilent E5070B/E5071B ENA Series RF Network Analyzers User's Guide," 2nd ed. Agilent Technol., Palo Alto, CA, 2003.

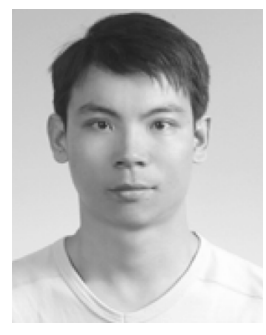

Kun-Tzu Chen was born in Changhua, Taiwan, R.O.C., on January 9, 1975. He received the B.S. degree from the Tatung Institute of Technology, Taipei, Taiwan, R.O.C., in 1997, and is currently working toward the M.S. degree in communication engineering at National Chiao Tung University, Hsinchu, Taiwan, R.O.C.

His current research interests are microwave circuits, LTCC RF passive components, and LTCC front-end modules for wireless local area network (WLAN) applications.

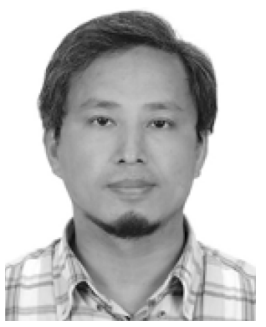

Shyh-Jong Chung (M'92-SM'06) was born in Taipei, Taiwan, R.O.C. He received the B.S.E.E. and Ph.D. degrees from National Taiwan University, Taipei, Taiwan, R.O.C., in 1984 and 1988, respectively.

Since 1988, he has been with the Department of Communication Engineering, National Chiao Tung University, Hsinchu, Taiwan, R.O.C., where he is currently a Professor. From September 1995 to August 1996, he was a Visiting Scholar with the Department of Electrical Engineering, Texas A\&M University, College Station. His research interests include the design and application of active and passive planar antennas, LTCC-based RF components and modules, packaging effects of microwave circuits, vehicle collision warning radars, and communications in intelligent transportation systems (ITSs).

Dr. Chung served as the treasurer of the IEEE Taipei Section (2001-2003) and the chairman of the IEEE Microwave Theory and Techniques Society (IEEE MTT-S) Taipei Chapter (2005-2007). He was the recipient of the 2005 Outstanding Electrical Engineering Professor Award of the Chinese Institute of Electrical Engineering and the 2005 Teaching Excellence Award of National Chiao Tung University. 